British Journal of Psychiatry (1994), 165, 551-559

\title{
Correspondence
}

Contents: Post-natal psychiatric morbidity/Head circumference at birth and schizophrenia/Lithium mutagenicity/High-dose antipsychotic medication/ Sinus bradycardia due to fluvoxamine overdose/ Treatment of drug-induced anorgasmia/Violence in psychiatric units/Male erotomania and dangerousness/Outdated ECT machines/Cognitive function and fall-related fractures/Creativity and psychopathology/Lack of care in Rwanda/Cognitive therapy in panic disorder

\section{Post-natal psychiatric morbidity}

SIR: Ballard et al (BJP, June 1994, 164, 782-788) assert that their data "supports the work of Cooper et al (1988) and O'Hara et al (1984) that there is no additional vulnerability to depression in post-natal mothers above that in a control group".

This conclusion is not supported. Using their results, the $95 \%$ confidence interval for the excess psychiatric morbidity in post-natal mothers at six weeks is $(-1.3,19.6)$. This interval includes the point zero, so it is compatible with the "null hypothesis" that there is no excess morbidity in the post-natal group; yet it is equally compatible with a true excess morbidity of nearly $20 \%$.

Looked at another way, the statistical power of this study is only $37 \%$, meaning that there is a $63 \%$ probability of a type II error-accepting the "null hypothesis" when it is false. To increase the power to $80 \%$ would require a sample size of at least 343 subjects in each group. For the fathers, the power of the study is only $15 \%$, and to increase this to $80 \%$ would require a sample size of at least 1068 subjects in each group.

Also, neither of the studies quoted investigated the prevalence of depression in a control group. They used comparative data from community surveys carried out by other investigators.

Finally, the conclusion of O'Hara et al's paper was that "The $12 \%$ prevalence of postpartum depression reported here might have been about double the rate of depression in community women, using criteria identical to ours. Consistent with reports of higher rates of psychiatric hospitalization for puerperal than for non-puerperal women, it appears that our subjects experienced a higher rate of depression than did non-puerperal women." This conclusion is, therefore, exactly the opposite of what Ballard et al state it to be.

Cooper, P. J., CAmprell, E. A., DAy, A., et al (1988) Nonpsychotic psychiatric disorder after childbirth: a prospective study of prevalence, incidence, course and nature. British Journal of Psychiatry, 152, 799-806.

O'Hara, M. W., Neunaber, D. J. \& Zekoski, E. M. (1984) Prospective study of post-partum depression: prevalence, course and predictive factors. Journal of Abnormal Psychology, 93, 158-171.

Bill Plummer

UMDS

London SE1 9RT

\section{Head circumference at birth and schizophrenia}

SIR: McNeil et al (BJP, April 1993, 162, 517-523) report that head circumference (HC) per se and the ratio of it to body length (BL), at the time of birth in preschizophrenics are significantly smaller when compared with control neonates. This supports the neurodevelopmental hypothesis of schizophrenia (Murray \& Lewis, 1987; Waddington, 1993).

In Japan, each pregnant woman has, since 1948, been officially issued with a "Maternal and Child Health Handbook" (MCHH) ("Boshi-KenkouTechou" in Japanese). Obstetricians are obliged to fill in the MCHH with regard to the progress of pregnancy and delivery, and each baby is followed up by paediatricians or other care professionals for several years. Most mothers keep their MCHHs well after their children become adults. We collected 64 MCHHs of DSM-III-R (American Psychiatric Association, 1987) schizophrenic patients ( 34 men) born between 1959 and 1979, who were treated at the psychiatric clinics of Teikyo University Hospital and three associated hospitals, in the Tokyo area, between April 1993 and March 1994. We also obtained $45 \mathrm{MCHHs}$ of their healthy siblings (22 men) born between 1961 and 1985 .

The mean (s.d.) $\mathrm{HC}$ at birth in preschizophrenics and in their siblings were 33.1 (1.6) and $33.4(1.3) \mathrm{cm}$ respectively. The ratios (s.d.) of $\mathrm{HC}$ to $\mathrm{BL}$ at birth were $0.661(0.034)$ for preschizophrenics 